\title{
Aportes para el debate. Instrumentos de gestión ambiental en la provincia de Buenos Aires, Argentina. Una mirada interdisciplinar
}

Contributions for Discussion. Environmental Management Instruments in Buenos Aires Province, Argentina. An Interdisciplinary Approach Aportes para o debate. Instrumentos de gestão ambiental na província de Buenos Aires, Argentina. Uma olhada interdisciplinar

\author{
Clara María MinaVerRY* \\ VERÓNICA LUCÍA CÁCERES $S^{* *}$
}

FECHA DE RECEPCIÓN: 06 DE ENERO DE 2015. FECHA DE ACEPTACIÓN: 28 DE AGOSTO DE 2015

Doi: dx.doi.org/10.12804/esj18.01.2016.02

Para citar este artículo: Minaverry, C. y Cáceres, V. (2016). Aportes para el debate. Instrumentos de gestión ambiental en la provincia de Buenos Aires, Argentina. Una mirada interdisciplinar. Estudios Socio-Jurídicos, 18(1), 57-78. Doi: dx.doi. org/10.12804/esj 18.01.2016.02

\section{RESUMEN}

El artículo recupera reflexiones de dos investigaciones que siguieron una metodología cualitativa. Contribuye al conocimiento sobre los instrumentos de gestión ambiental en una jurisdicción particular de Argentina: la provincia de Buenos Aires (PBA). Tiene como punto de partida el reconocimiento de la importancia del trabajo interdisciplinar en la reflexión sobre la problemática ambiental y centra la atención por un lado, en las herramientas de gestión ambiental propuestas en dos campos de las Ciencias Sociales: la Economía Ambiental y el Derecho Ambiental y, por otro, presenta una caracterización y un análisis de los principales instrumentos de gestión ambiental en la jurisdicción señalada. El análisis permite señalar que los instrumentos tienen escasa implementación, porque las agencias que desarrollan las funciones ambientales no tienen capacidad técnica y son poco conocidas por la sociedad civil.

Palabras clave: Derecho Ambiental, Economía Ambiental, sustentabilidad, provincia de Buenos Aires.

* Investigadora del Consejo Nacional de Investigaciones Científicas y Técnicas (CONICET) y del Instituto de investigaciones jurídicas y sociales Ambrosio Gioja, Facultad de Derecho, Universidad de Buenos Aires, Docente e investigadora de la Universidad Nacional de Luján y de la Universidad Tecnológica Nacional, Argentina. Correo electrónico: cminaverry@derecho.uba.ar

** Docente e investigadora del Instituto de Desarrollo Humano (Área Economía) de la Universidad Nacional de General Sarmiento, Argentina. Correo electrónico: vcaceres@ungs.edu.ar 


\section{ABSTRACT}

This paper recovers reflections of two studies that followed a qualitative methodology. It contributes to knowledge about environmental management instruments in a particular jurisdiction of Argentina, the Buenos Aires Province (BAP). It has as its starting point, the recognition of the importance of interdisciplinary work in reflection on environmental issues. It focuses the attention, on one hand, in the environmental management tools proposed in two fields of social sciences: environmental economics and environmental law. On the other hand, it presents a characterization and analysis of the environmental main instruments of management in the indicated jurisdiction. The analysis allows to point out that the instruments has a poor implementation because the agencies that develop environmental functions do not have a technical capacity and it is little known by the civil society.

Keywords: Environmental Law, environmental economics, sustainability, Buenos Aires province.

\section{RESUMO}

O artigo recupera reflexões de duas investigações que seguiram uma metodologia qualitativa. Contribui ao conhecimento sobre os instrumentos de gestão ambiental em uma jurisdição particular da Argentina, a Província de Buenos Aires (PBA). Tem como ponto de partida o reconhecimento da importância do trabalho interdisciplinar na reflexão sobre a problemática ambiental. Centra a atenção por um lado, nas ferramentas de gestão ambiental propostas em dois campos das Ciências Sociais: a economia ambiental e o direito ambiental. Por outro, apresenta uma caracterização e análise dos principais instrumentos de gestão ambiental na jurisdição assinalada. A análise permite assinalar que os instrumentos têm escassa implementação porque as agências que desenvolvem as funções ambientais não têm capacidade técnica e são pouco conhecidas pela sociedade civil.

Palavras-chave: Direito ambiental, economia ambiental, sustentabilidade, província de Buenos Aires. 


\section{Introducción}

Desde los primeros años de la década del setenta, la cuestión ambiental emergió en la agenda internacional y en distintos espacios comenzó a discutirse sobre el deterioro, la contaminación y el agotamiento de los recursos naturales y del ambiente en su conjunto. En paralelo, la cuestión se incorporó como objeto de estudio a distintas disciplinas y existe cierto consenso en que, por las distintas aristas involucradas, su abordaje demanda una mirada interdisciplinar que considere aspectos técnicos, sociales, económicos, ecológicos, políticos y legales.

La Economía y el Derecho son disciplinas de las Ciencias Sociales, en la que la discusión sobre el ambiente ha adquirido relevancia en los últimos años.

Con el interés de contribuir al debate sobre la política ambiental y, en particular, sobre los instrumentos de gestión ambiental, desde una mirada interdisciplinar, el presente artículo centra la atención en una jurisdicción particular de Argentina: la PBA.

Cabe señalar que la PBA es la principal provincia argentina, país que adopta constitucionalmente una forma federal de gobierno, que reconoce la prexistencia de las provincias a la Nación y, por ello, estas conservan las competencias no delegadas al Gobierno federal. Las veintitrés provincias, incluidas la PBA y la CABA, conforman instancias autónomas que dictan sus propias Constituciones y votan por sus gobernantes. En materia ambiental, desde la reforma constitucional de 1994, le corresponde a la Nación la responsabilidad de "dictar las normas que contengan los presupuestos mínimos de protección" y a las provincias, que tienen "el dominio originario de los recursos naturales existentes en su territorio", complementarlos (Artículos 41 y 124 de la Constitución Nacional). ${ }^{1}$

La reforma constitucional significó un hito histórico en lo relativo a la evolución del Derecho Ambiental, ya que incorporó en el Capítulo Segundo de la parte dogmática, titulada "Nuevos derechos y garantías", en el Artículo 41, el derecho a un ambiente sano, equilibrado y apto para el desarrollo humano, cuyas características son la concepción derecho-deber

La Nación ejerce jurisdicción sobre la navegación, el comercio interprovincial e internacional, las relaciones internacionales y la celebración de tratados internacionales. 
de un ambiente sano en cuanto a su exigibilidad y participación; el compromiso intergeneracional de preservación del ambiente para las generaciones presentes y futuras; la recepción de la noción de desarrollo sustentable; apto para el desarrollo humano; la jerarquía constitucional de la obligación de recomponer el daño ambiental. Asimismo, añadió, con rango constitucional, pactos y convenciones como el Pidesc, que reconoce un conjunto de derechos vinculados de manera estrecha con el derecho al ambiente como la calidad de vida, la salud y el desarrollo.

Esto tuvo su correlato en la Constitución de la PBA que, en su Artículo 28, planteó:

[...] los habitantes de la Provincia tienen el derecho a gozar de un ambiente sano y el deber de conservarlo y protegerlo en su provecho y en el de las generaciones futuras. La Provincia ejerce el dominio eminente sobre el ambiente y los recursos naturales de su territorio incluyendo el subsuelo y el espacio aéreo correspondiente, el mar territorial y su lecho, la plataforma continental y los recursos naturales de la zona económica exclusiva, con el fin de asegurar una gestión ambientalmente adecuada [...].

El ente provincial asume un conjunto de funciones ambientales relevantes que incluyen la preservación, recuperación y conservación de los recursos naturales renovables y no renovables, el control del impacto ambiental, la planificación del uso racional de los recursos y la promoción de actividades que eviten la degradación del aire, de los recursos hídricos, de los suelos, entre otras (Cáceres, 2014).

Más allá de ello, los instrumentos de gestión ambiental implementados por las provincias han sido poco explorados y sobre la PBA existen escasos antecedentes.

El artículo recupera reflexiones de dos investigaciones: la primera, en el área de Economía, del Instituto de Desarrollo Humano de la Universidad Nacional de General Sarmiento, denominada "Enfoques económicos y representaciones sociales de la problemática ambiental desde una perspectiva crítica" y la segunda, en el Departamento de Ciencias Sociales de la Universidad Nacional de Luján, titulada "La relación entre la protección de los recursos hídricos y de los bosques. Situación legal en Argentina y en Uruguay". 
Ambas investigaciones adoptaron una metodología cualitativa, se valieron del método de observación documental, en especial, de la observación directa de fuentes secundarias, que incluyó el análisis de documentos de organismos públicos, de la normativa obligatoria y voluntaria de Derecho Ambiental y de Derecho Administrativo y los instrumentos de gestión vigentes para la PBA.

El artículo se estructura de la siguiente forma: tras la presente introducción, se identifican y caracterizan las herramientas de gestión ambiental propuestas desde la Economía Ambiental y el Derecho Ambiental; luego, se centra la atención en los principales instrumentos de gestión ambiental previstos en la PBA y, por último, se ofrecen algunas conclusiones.

\section{Herramientas de gestión ambiental desde la Economía Ambiental y el Derecho Ambiental}

A partir de la década del setenta del siglo XX, se observa que el tratamiento de la problemática ambiental se instaló en la agenda internacional como un asunto a ser atendido en virtud de dos procesos íntimamente vinculados: el creciente deterioro, el agotamiento y la mercantilización de los recursos naturales y la expansión de los conflictos socio ambientales (Cáceres, 2014).

La temática ha dado lugar al desarrollo de las siguientes conferencias de Naciones Unidas o Cumbres de la Tierra: la Conferencia de Río de 1992, de las Naciones Unidas sobre ambiente y desarrollo, en donde se emitió la Declaración de Río; la Cumbre Mundial sobre el Desarrollo Sostenible de Johannesburgo, de 2002, en donde se firmó el Plan de acción y la Declaración Política de Johannesburgo sobre el Desarrollo Sustentable y la Conferencia de las Naciones Unidas sobre el Desarrollo Sostenible "Río+20", celebrada en 2012, en donde se acordó la Resolución 66/288, titulada "El futuro que queremos".

Desde la Cumbre de 2002, los organismos internacionales BID, PNUMA y la UE, entre otros, han apelado "al multilateralismo y a la solidaridad intergeneracional como herramientas básicas para afrontar la erradicación de la pobreza, construir relaciones internacionales basadas en la equidad y la justicia, adaptar los patrones de consumo y producción con 
un enfoque sustentable" (Preiss y Roca, 2006, p. 16) y se ha establecido como paradigma que la sostenibilidad es:

[...] la base de cualquier modelo de desarrollo que se proponga, y se sugiere el tratamiento con el mismo grado de importancia de las variables económica, social y ambiental, lya que] de ello depende el equilibrio entre equidad social, crecimiento económico y sustentabilidad ambiental (Preiss y Roca, 2006, p. 16).

A la luz de las distintas Cumbres, comenzó a expandirse también un conjunto de herramientas de gestión ambiental que integra aportes de distintas disciplinas. En este artículo nos interesa señalar las principales estrategias por dos campos de conocimiento: la Economía Ambiental y el Derecho Ambiental.

La Economía Ambiental, vinculada al marco epistemológico neoclásico, ocupa un lugar hegemónico, ${ }^{2}$ desde el cual se ha desarrollado una serie de métodos vinculados a los mecanismos de mercado, los costes marginales y los análisis de costo-beneficio para explicar los problemas ambientales y pensar posibles alternativas.

En el Derecho Ambiental - rama nueva del Derecho que se nutre de los principios de otras disciplinas y del Derecho Público, del Derecho Privado, del Derecho Internacional, etc.- también se encuentran distintos posicionamientos, sobre todo en torno al objeto material del concepto "ambiental". En este caso, hay una mirada que considera que "prácticamente todo" es ambiente, otra más restringida que enfatiza el ámbito de los

2 La conformación de la economía neoclásica como paradigma, enfoque, programa o visión puede ubicarse a partir de las obras de tres economistas que pertenecían a ámbitos intelectuales diversos, heterogéneos y con poco intercambio académico para la época. Por un lado, la obra de Karl Menger (1840-1921), Principios de economía política (1871), surgida en Viena; la obra de William Jevons (1935-1882), Theory of Political Economy (1871), escrita en la ciudad industrial de Manchester y la obra de León Walras (1934-1910), Elementos de Economía Política pura (1874), en Lausana (Suiza). De acuerdo con Forcinito, (2010), el paradigma neoclásico se sostiene sobre la naturalización del orden de cosas inherente al sistema capitalista, como las instituciones mercado y Estado-Nación; la centralidad del sistema de precios del mercado competitivo para la asignación eficiente de recursos; la resolución armónica de los conflictos por parte del mercado gracias a la libertad de elección individual (soberanía del consumidor); la sociedad como suma de los comportamientos individuales (individualismo metodológico) y la negación de una sociedad dividida en clases; la idea de individuos concebidos atomísticamente y portadores de una racionalidad utilitarista (homo economicus); la determinación del carácter técnico (y no social o vinculado a la estructura de poder) de la distribución del ingreso entre los factores de producción. 
bienes comunes (el agua, el aire y los procesos de contaminación que los afecta) y una intermedia que incluye: a) los recursos naturales y su uso; b) los accidentes naturales, y c) la problemática de los asentamientos humanos (Menéndez, 2014).

La Economía Ambiental y el Derecho Ambiental han hecho aportes al estudio y a la implementación de los instrumentos de gestión de regulación directa como los estándares ambientales. Entre ellos se encuentran: los de calidad ambiental (que establecen el grado de concentración de sustancias físicas, biológicas o químicas presentes en aire, agua o suelo, en su estado de cuerpo receptor, que no representan riesgo significativo o severo para la salud o el ambiente); de emisión (que fijan el grado máximo de emisiones realizadas); tecnológicos (que acuerdan el tipo de equipamiento para tratar las emisiones antes de la descarga); de productos y de insumos (que definen el tipo de insumo que se puede utilizar o determina la cantidad máxima de insumos potencialmente contaminantes que pueden incluirse en cada proceso de producción) (Argerich, 2009).

Asimismo, se ha contribuido a la definición de los impuestos o tasas ambientales, basadas en los aportes de Pigou (1950), para quien el cobro de impuestos era una forma de asumir las externalidades. ${ }^{3}$

Los instrumentos de gestión ambiental más utilizados tienen un carácter de regulación directa y apuntan a la prevención, es decir, procuran favorecer la concientización y el desarrollo de prácticas de respeto por la naturaleza.

Entre los instrumentos se destacan:

- La sanción de normativa que regula el uso de los recursos naturales.

- Los estudios de impacto ambiental que contienen análisis técnicos y científicos para, al menos, identificar, predecir, interpretar y comunicar los impactos, tanto positivos como negativos, que puedan producir las acciones sobre el ambiente. Deben efectuarse de forma previa a la implementación de proyectos.

- La aplicación de normas voluntarias y certificación ambiental que no solo contribuyen a cumplir con las exigencias legislativas, sino que

3 Cuando el impuesto fuera igual al coste externo marginal, en lo que se considera el grado aceptable de contaminación. 
además permiten controlar y minimizar el impacto de los elementos o actividades susceptibles de producir daños sobre el ambiente.

- La contratación de la póliza del seguro de caución ambiental, incluidas pólizas por el daño causado por las actividades productivas, ya sea por accidentes o hechos súbitos, etc.

- La investigación ambiental básica y tecnológica vinculada a distintos sectores económicos.

- La educación formal e informal con la inclusión de contenidos en los diseños curriculares sobre ambiente, territorio y economía, así como la formación de técnicos y especialistas, entre otros.

A continuación se caracterizan las herramientas de gestión ambiental vigentes en la PBA.

\section{Gestión ambiental en la provincia de Buenos Aires}

\subsection{Consideraciones preliminares}

La PBA se ubica en el centro este del país y tiene una extensión que equivale a un $11,1 \%$ del territorio argentino. En 2010 contaba con una población que ascendía a 15.625.084 habitantes, lo que representaba un $38,95 \%$ del total nacional, distribuido de forma heterogénea en dos regiones geográficas diferenciadas con dinámica demográfica propia: el Gran Buenos Aires o conurbano bonaerense, integrado por veinticuatro partidos que rodean a la CABA y concentraban un $63,46 \%$ de la población y el interior provincial, conformado por 111 partidos, en donde se localizaba el resto de la población (Instituto Nacional de Estadísticas y Censos, 2010).

En materia ambiental, los problemas que afectan a la provincia incluyen la creciente generación de residuos y efluentes que contaminan el agua superficial y subterránea, la ausencia de infraestructura sanitaria en una parte relevante del territorio, que ocasiona el uso de alternativas menos eficientes (solo 47,57\% de los hogares accedía al servicio de cloacas en 2010), la contaminación industrial producida por los emprendimientos instalados en las riberas y el poco tratamiento de los efluentes domésticos recolectados por 
las empresas prestatarias del servicio de cloacas. Estas fuentes han generado graves problemas de contaminación de algunas cuencas hidrográficas. ${ }^{4}$

La institucionalidad responsable de la gestión ambiental en la provincia resulta de distintos eventos, sobre todo de la década del noventa del siglo $\mathrm{XX}$, vinculadas, por un lado, a la sanción de la primera Ley del Ambiente del país, Ley 11723 y la incorporación de la temática a la Constitución provincial y, por otro, a la creación de organismos específicos para atender la cuestión. Isuani (2013) señala que la temática ambiental en la PBA estuvo relacionada, en principio, con el campo sanitario; de hecho, la Dirección Provincial de Medio Ambiente fue creada en 1991 y dependió de la Subsecretaría de Infancia, Familia y Medioambiente del Ministerio de Salud. En 1993, fue reemplazada por el Instituto Provincial de Medio Ambiente y estuvo en actividad hasta 1995, cuando se creó la Secretaría de Política Ambiental, dependiente directa de la Gobernación. En 2007, la Secretaría fue transformada en el OPDS, en el ámbito del Ministerio de Jefatura de Gabinete.

En la actualidad, el OPDS es el organismo rector de la política ambiental y tiene como funciones planificar, formular, proyectar, fiscalizar, ejecutar la política ambiental y preservar los recursos naturales; ejercer el poder de policía, establecer y fiscalizar el cumplimiento de la política sobre contaminación industrial, sus efluentes y del ambiente en general; intervenir en la conservación, protección y recuperación de reservas, áreas protegidas y bosques, en el uso racional y la recuperación de suelos; la promoción de actividades vinculadas con la educación ambiental y con los estudios de impacto ambiental, entre otras (Decreto del Poder Ejecutivo Provincial 23 de 2007).

El OPDS comparte funciones ambientales con un conjunto heterogéneo de actores estatales como la Autoridad del Agua, que lleva adelante la planificación hidrológica y la reglamentación, supervisión y vigilancia de todas las actividades y obras relativas al estudio, a la captación, al uso, a la conservación y evacuación del agua, etc.; el Organismo de Control del

4 A modo de ejemplo, la cuenca Matanza-Riachuelo es emblemática por los grados de contaminación. Desde mediados del siglo pasado, en esta se ubica el polo industrial y es competencia de tres jurisdicciones (el Estado nacional, el Estado provincial y el Estado de la CABA) que se encuentran condenados, junto con algunas empresas, por su contaminación y obligados por la justicia a implementar programas de saneamiento ambiental integral. 
Agua de la Provincia de Buenos Aires, que tiene a su cargo el control y la fiscalización de la prestación de los servicios de agua y cloacas, así como la defensa de los intereses de los usuarios; la intervención en las cuestiones relacionadas con la actividad de las prestatarias del servicio; velar por la protección del ambiente, etc.; la Corporación de Fomento de la Producción, que administra el servicio de riego del río Colorado en los partidos de Villarino y Patagones) y el Consejo Consultivo Cuenca Alta del Comité del río Reconquista, que tiene como objeto prestar servicios y tomar acciones conducentes a la gestión integral y preservación del recurso hídrico de la cuenca del río Reconquista.

Más allá de las funciones importantes que asume, el OPDS solo ejecuta una parte pequeña del gasto público provincial destinado al ambiente, por lo que tiene un papel casi formal en la política ambiental, ya que cuenta con escasos recursos financieros para implementar los programas ambientales asociados con la amplitud de las funciones (Cáceres, 2014). ${ }^{5}$

El OPDS, como el resto de las agencias que cumplen funciones ambientales, promueve instrumentos de gestión ambiental que otorgan importancia a la previsión, entre los cuales se encuentran: a) la evaluación de impacto ambiental; b) la aplicación de normas voluntarias y certificación ambiental, y c) la contratación de la póliza del seguro de caución ambiental.

\subsection{Principales instrumentos de gestión ambiental}

\subsubsection{La evaluación de impacto ambiental}

El Estado provincial tiene que garantizar que los habitantes gocen "de un ambiente sano, adecuado para el desarrollo armónico de la persona", de acceso "a la información vinculada al manejo de los recursos naturales que administre el Estado", de participación de los procesos referentes al "manejo de los recursos naturales y la protección, conservación, mejoramiento y restauración del ambiente" y soliciten "a las autoridades de adopción de medidas tendientes al logro del objeto de la presente Ley, y a denunciar el incumplimiento de la misma" (Artículo 2, Ley Integral del medioambiente y de los recursos naturales, Ley 11723). En este contexto, la evaluación

5 Para un análisis de la evaluación del gasto ambiental en la PBA, véase Cáceres (2014). 
de impacto ambiental es un procedimiento técnico-administrativo obligatorio para los nuevos proyectos que procuren ser radicados en la PBA e impliquen acciones $u$ obras susceptibles de producir efectos negativos sobre el ambiente o sus elementos. Se realiza de forma multidisciplinaria e interdisciplinaria y su desarrollo tiene que aportar información para la toma de decisiones. Se presenta en la Dirección Provincial de Evaluación de Impacto Ambiental dependiente de la OPDS y queda incorporada como un documento exigido por la autoridad competente, antes del desarrollo de actividades. Incluye no solo a las actividades privadas, sino a las cometidas por organismos estatales como las obras de agua y saneamiento, hidráulicas, electrificación de ferrocarriles, paso en bajo nivel, etc.

La Ley Provincial 11459 de radicación industrial también establece que "todos los establecimientos industriales deberán contar con el pertinente Certificado de Aptitud Ambiental como requisito obligatorio indispensable para que las autoridades municipales puedan conceder, en uso de sus atribuciones legales, las correspondientes habilitaciones industriales" (Artículos 3 y 7) y plantea que el Certificado de Aptitud Ambiental será expedido por la autoridad de aplicación o el municipio, según corresponda, previa evaluación ambiental y de su impacto en la salud, la seguridad y los bienes del personal y de la población circundante.

Con este instrumento probabilístico se puede lograr que los costos preventivos sean menores que los costos correctivos y se involucre a la sociedad civil. Una aproximación a sesenta estudios presentados y evaluados por la OPDS en cuatro municipios, durante 2009 y 2014, muestra que el grueso de las obras para el conurbano bonaerense (municipios de Quilmes y San Martín) fue efectuado por actores estatales y en el interior provincial (Bahía Blanca y General Pueyrredón) por empresas privadas y, en menor medida, por organismos públicos. El centro de las propuestas en el conurbano se relaciona con agua, saneamiento y desagües pluviales. Estas son áreas en donde el Estado tiene alta participación desde la oleada de reestatizaciones de las prestatarias de los servicios e intentan dar respuestas al déficit en el acceso a los servicios que se observa en la zona (fueron presentadas por Aguas y Saneamientos Argentinos S. A. y la Dirección Provincial de Hidráulica, entre otros). En el caso del interior, las obras estaban destinadas a mejorar la producción y distribución de energía eléctrica, campos de acción bajo gestión privada. 
Una de las críticas centrales a este instrumento es que es solicitado a especialistas de consultoras privadas que, de acuerdo con los propios involucrados, podrían asumir distintos sesgos y cuentan con escasa participación de la sociedad civil.

Sin embargo, el procedimiento administrativo de la evaluación de impacto ambiental exige que se celebre una audiencia pública obligatoria en donde participan los interesados en el proyecto pertenecientes a la comunidad. Las recomendaciones emanadas de la misma no son vinculantes, pero deben ser tenidas en cuenta cuando la autoridad administrativa emita la declaración de impacto ambiental.

Resulta fundamental que la publicación de la audiencia pública y el tiempo destinado a realizarla sean adecuados, con el fin de que la mayor cantidad de personas pueda participar de la misma y hacer sus aportes.

Las organizaciones sin fines de lucro (ONG) también cumplen un rol fundamental en la defensa de los intereses de los ciudadanos; en este caso, de todos los vinculados con los derechos humanos y en relación con la protección del ambiente.

Gráfico 1. Evaluaciones de impacto ambiental como fueron presentadas por organismos públicos o empresas privadas

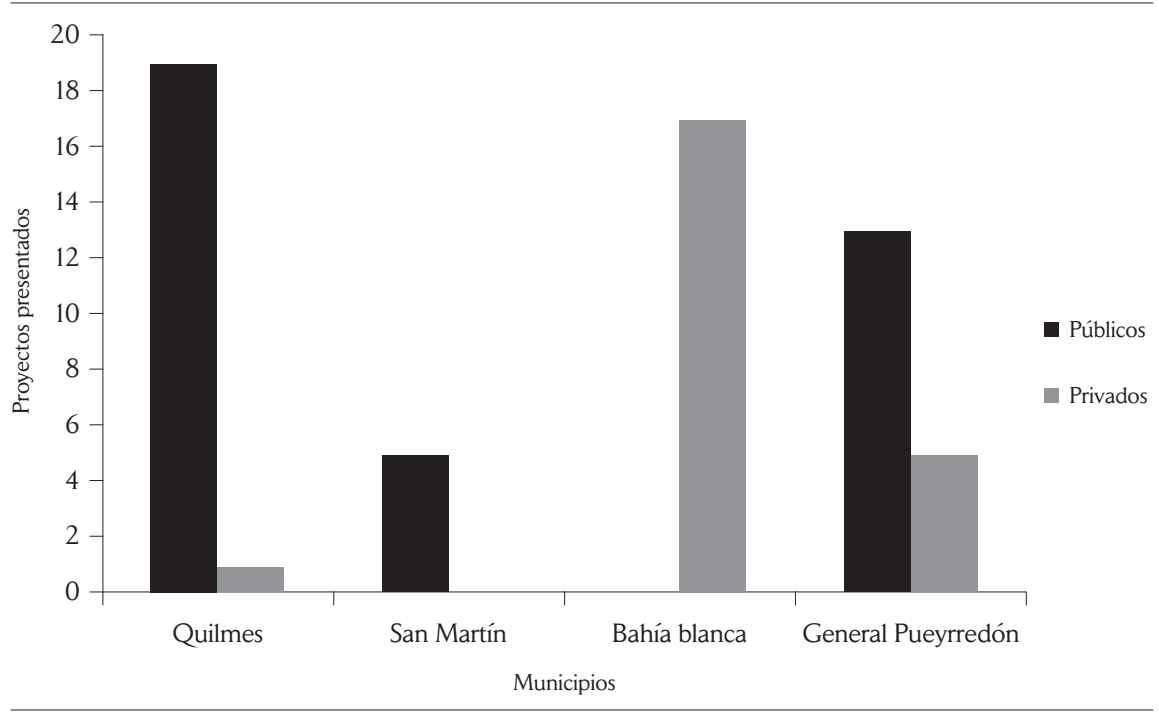

Fuente: elaboración propia con base en la información de la OPDS. 


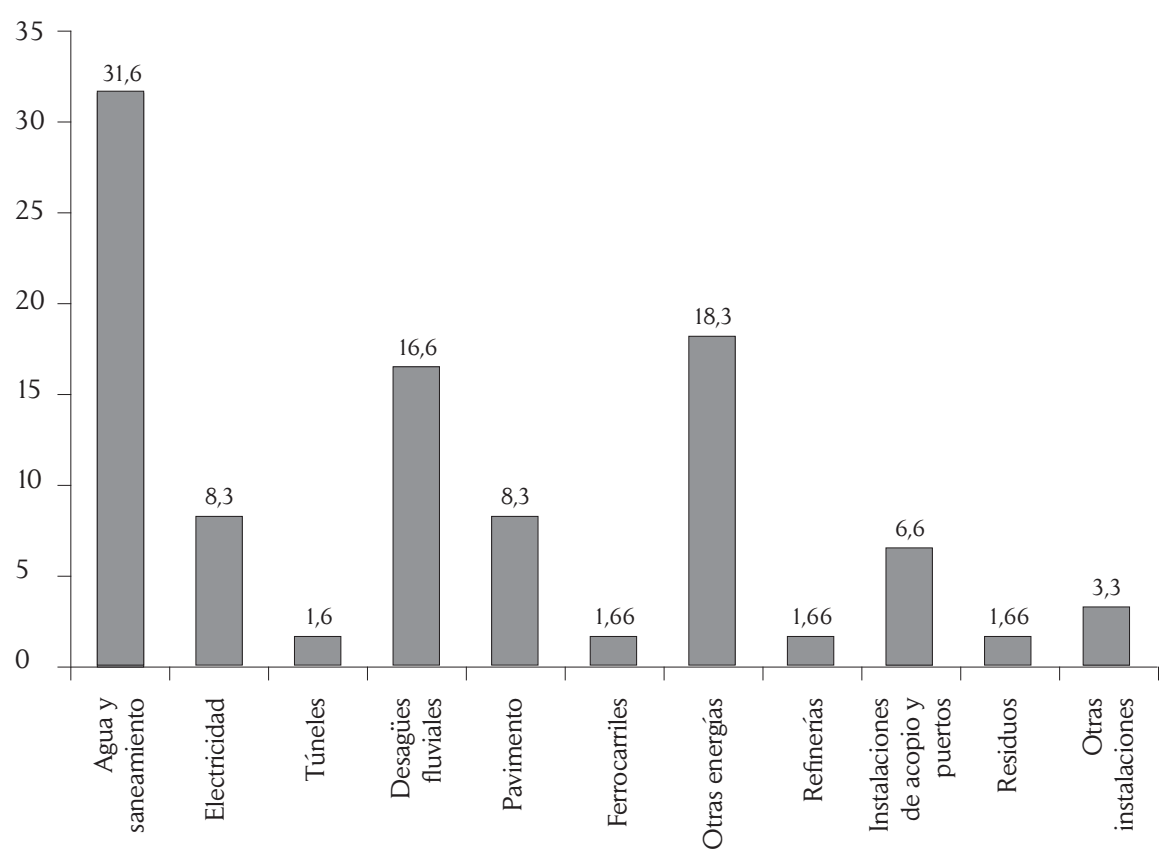

Fuente: elaboración propia con base en la información de la OPDS.

\subsubsection{La aplicación de normas voluntarias y certificación ambiental}

Otro instrumento de gestión ambiental se refiere a la implementación de un sistema de gestión ambiental que incluya la aplicación de normas voluntarias y la certificación ambiental. Para las empresas que operan en suelo bonaerense, estos suponen una serie de ventajas: por un lado, reducen los costos de contratación del seguro ambiental y, por otro, tienden a considerarse indicadores de la actitud sustentable en el momento de registrar inspecciones ambientales.

Se entiende que la aplicación de una norma internacional de carácter voluntario, como la serie ISO 14.000, contribuye a delinear objetivos ambientales y metas profesionales de forma periódica (con base en los aspectos ambientales significativos de las actividades de las empresas).

La posibilidad de certificar un sistema de gestión ambiental conlleva un esfuerzo para los integrantes de las organizaciones que participan en esta tarea, sobre todo durante las fases de diseño e implantación del sistema. 
En la PBA, las normas de gestión tienen como requisitos: mantener una política y unos objetivos ambientales destinados a la mejora continua de sus actuaciones frente al ambiente; hacer una comprobación de su situación respecto a la legislación y normativa ambiental aplicable a sus actividades, productos y servicios, para garantizar su cumplimiento; designar y documentar funciones, responsabilidades y recursos para el cumplimiento de sus prioridades; identificar y valorar los aspectos ambientales asociados con sus actividades, productos y servicios y definir pautas de actuación para su prevención y control; avalar la formación de su personal en materia de gestión ambiental y establecer mecanismos de comprobación para asegurar el cumplimiento de la política ambiental de la organización.

Cuando la empresa haya implementado el sistema de gestión, debe proceder a la certificación del mismo (dar validez frente a terceros) y, para eso, se necesita una entidad de certificación que audite el sistema y lo certifique. Previamente, la empresa debe cumplir con uno de los puntos y requisitos que recoge la norma voluntaria y aplicarlos al desarrollo de sus actividades.

Para cumplir todos los requisitos de la misma, se puede contratar un servicio externo que haga todos los trabajos anteriores a la implantación, así como la implementación propiamente dicha o puede realizarlo la propia empresa. Por lo general, se suelen contratar los servicios de consultoras especializadas.

Cuando la empresa certifica la norma, la consultora que la estableció deberá hacer auditorías periódicas, con el fin de efectuar un seguimiento del grado de cumplimiento de las exigencias definidas por la empresa. Además, deberá indicarle los incumplimientos (en caso de existir) y exigir su cumplimiento, tendiente a lograr la mejora continua y la aplicación del principio de desarrollo sustentable a cualquier actividad que desempeñen.

\subsubsection{La contratación de la póliza del seguro de caución ambiental}

El seguro ambiental es otro instrumento de gestión ambiental. Se trata de una garantía financiera exigible a toda persona física o jurídica, pública o privada, que desarrolle actividades riesgosas para el ambiente, los ecosistemas y sus elementos constitutivos.

En Argentina, de acuerdo con la Ley General del Ambiente, Ley 25675, la contratación del seguro ambiental es imperativa. Asimismo, en la PBA, la Ley Provincial 11459 acordó la obligatoriedad de contar con seguro am- 
biental para todas las industrias que se instalen, amplíen o modifiquen sus establecimientos o explotaciones dentro de la jurisdicción provincial.

Las pólizas de seguro por daño ambiental aceptadas por el OPDS deben ser emitidas por las compañías de seguro aprobadas por la Superintendencia de Seguros de la Nación y la Secretaría de Ambiente y Desarrollo Sustentable de la Nación y, a su vez, deben ser compañías que hayan acreditado su capacidad y factibilidad operativa para cometer acciones de recomposición del ambiente dañado (Resolución OPDS 165/10, modificada a su vez por la Resolución 186/12 del 28/09/2012).

La Resolución manda a los siguientes sujetos a contratar el seguro ambiental:

- Los establecimientos instalados en jurisdicción provincial clasificados de acuerdo con la Ley 11459 y su reglamentación, a los que corresponde la segunda categoría cuando su grado de complejidad ambiental sea igual o mayor a 14,5 puntos, conforme a las previsiones del Artículo 1 de la Resolución 481/11 de la Secretaría de Ambiente y Desarrollo Sustentable de la Nación.

- Los establecimientos a los que corresponde la tercera categoría, conforme a su grado de complejidad ambiental en los términos de la Ley 11459 y su reglamentación.

- Las personas físicas o jurídicas responsables de generación, tratamiento, almacenamiento y disposición final de residuos especiales, conforme a la Ley Provincial 11720.

La Resolución mencionada estableció que el OPDS puede exigir el cumplimiento de la obligación contenida en el Artículo 22 de la Ley General del Ambiente a aquellos establecimientos cuyo grado de complejidad ambiental sea inferior a 14,5 puntos, conforme al Artículo 1 de la Resolución 481/11 de la Secretaría de Ambiente y Desarrollo Sustentable de la Nación, cuando situaciones especiales así lo justifiquen, como vulnerabilidad del sitio de emplazamiento del establecimiento, antecedentes de desempeño ambiental, antigüedad y ubicación de depósitos de sustancias especiales u otros criterios específicos de riesgo ambiental.

Una de los mayores críticas que presentaba el sistema anterior era que brindaba tan solo una opción de aseguramiento - el de seguro de caución 
por daño ambiental-, comercializado de hecho por un número pequeño de compañías aseguradoras. Esta clase de seguro no transfería el riesgo a la compañía aseguradora, al estipularse que las tareas de remediación debían ser llevadas a cabo hasta cubrir la suma asegurada y luego la aseguradora podía repetir lo pagado frente al asegurado.

Este esquema no era viable en términos económicos, ya que los asegurados, además de ser directamente responsables de correr con la totalidad de los gastos de la recomposición, estaban obligados a contratar la prima.

El régimen que incorpora el Decreto 1638/12 trajo nuevas opciones de instrumentos, pues aparte de un seguro de caución por daño ambiental de incidencia colectiva, prevé la contratación de un seguro de responsabilidad por daño ambiental de incidencia colectiva (Artículo 1), que sí cumpliría con la transferencia del riesgo.

Dicho sistema no solo brinda otra opción de aseguramiento, sino que tampoco descarta la posibilidad del autoseguro, al no desechar el total del riesgo. Toda empresa podrá hacer una reserva por el daño no cubierto. Esta norma, sin duda, marcaba el camino hacia un conjunto de alternativas de contratación, así como opciones de remediadores para distintos tipos de actividades riesgosas con diferente grado de complejidad ambiental y capacidad empresarial.

Por su parte, la Corte Suprema de Justicia de la Nación, con fecha 11 de diciembre de 2014, se ha pronunciado en la causa "Recurso de hecho deducido por la demandada en la causa Fundación Medio Ambiente c/ EN-PEN- dto. 1638/12-SSN-resol. 37.160 s/medida cautelar autónoma", para revocar una medida cautelar dictada por la Cámara Nacional de Apelaciones en lo Contencioso Administrativo Federal, que había suspendido la vigencia de las normas reglamentarias del seguro ambiental, por las cuales se podían contratar dos tipos de seguros: uno de caución por daño ambiental de incidencia colectiva y uno de responsabilidad por daño ambiental de incidencia colectiva (Decreto 1638/12 y Resolución 37.160/12 de la Superintendencia de Seguros de la Nación).

La Corte ordenó que el expediente fuera remitido otra vez a la Cámara para el dictado de un nuevo fallo que contara con fundamentos razonados que lo sostuvieran como un acto constitucionalmente válido. A partir de esta decisión de la Corte, en principio, volvería a cobrar vigencia el Decreto 1638. 
Sin embargo, es importante tener en cuenta que la SAyDS dictó la Resolución 999/2014, que estableció - en línea con lo decidido por la Cámara en la sentencia que fue dejada sin efecto por la Corte- que las aseguradoras deben cumplir con ciertos requisitos para comercializar el seguro ambiental, entre ellos:

a) La acreditación de "capacidad técnica", mediante contratos con remediadores u operadores de residuos peligrosos.

b) La obtención de la conformidad ambiental previa.

c) Otros requisitos, como acreditar determinada solvencia patrimonial. Esta misma Resolución estableció un plazo de noventa días hábiles para que las compañías se readecúen a los nuevos requisitos.

En síntesis, de acuerdo con lo establecido por la Superintendencia de Seguros de la Nación, se considera que el Decreto 1638/12 y la Resolución SSN 37.160/12 están suspendidos.

Otra de las grandes discusiones se relaciona con la necesidad o no de establecer un límite para la remediación de tornar factible la implementación de seguros y, en todo caso, el tipo de límite que podría admitirse.

El sector industrial y de servicios critica la amplitud de la noción de recomposición que propone la LGA, al demandar el restablecimiento del ambiente al estado anterior a la producción del daño, porque torna la obligación excesivamente onerosa y de imposible cumplimiento.

En este sentido, creemos que la solución, en términos pragmáticos, no puede significar una interpretación restrictiva del concepto de daño ambiental o de recomposición que trae la LGA, por cuanto podría afectar la tutela constitucional del bien jurídico que se busca proteger.

En esta clase de seguros se incorporan novedosos aspectos de valoración económica (externalidades ambientales), muchas veces ausentes en el sistema tradicional de seguros.

Sin perjuicio de ello, también resulta de vital importancia que el tipo de control ambiental, conformidad u otro que se añada a la lógica de aseguramiento sea establecido con transparencia, proponga reglas claras y unívocas y defina procedimientos, parámetros y criterios objetivos para que sirva, a la vez, a fines preventivos y disuasivos. En ese sentido, se ha dicho que el seguro de caución, como está establecido en la normativa vigente, no 
obra como incentivo para la prevención de daño ambiental, como debería funcionar, a la luz del principio de prevención.

\section{Consideraciones finales}

En los últimos años, la problemática ambiental se ha instalado con fuerza en la agenda internacional y local, lo que da lugar a desarrollos teóricos y prácticos en el campo de las Ciencias Sociales.

En este artículo hemos enfatizado en los instrumentos de gestión ambiental propuestos desde dos campos particulares: la Economía Ambiental y el Derecho Ambiental, a partir de los cuales hemos caracterizado los instrumentos vigentes en la PBA, la jurisdicción más importante de Argentina.

El análisis nos permite señalar que, aunque en los últimos años han proliferado los instrumentos de gestión acordes con el principio de prevención, su implementación a cargo de un organismo con amplias funciones y escasos recursos conforma un serio limitante.

Además, las normativas conocidas por la sociedad civil no contribuyen con eficiencia al reclamo por la exigibilidad de derechos básicos vinculados a la protección del ambiente.

Otros instrumentos, como los impuestos o tasas ambientales, aún no logran institucionalizarse. Hace poco, el Estado provincial ha intentado efectuar el cobro de un canon por el uso del agua, previsto en su Código de Agua de 1999. Los impuestos o tasas son bastante resistidos por aquellos actores económicos que utilizan los recursos naturales como factores medulares de la producción.

En la actualidad, existen voces críticas respecto a la eficacia de las herramientas de gestión ambiental vigentes en la PBA, como a la relevancia, por ejemplo, de las normas voluntarias que complementan a las obligatorias y vigentes en un determinado lugar (Minaverry y Gally, 2013).

Uno de los ejes que se cuestiona en la evaluación de impacto ambiental es el sesgo que pueden tener, en tanto son solicitadas por los interesados a empresas privadas y en la que la participación de actores de la sociedad civil no está contemplada.

Sin duda uno de los instrumentos de gestión más polémicos es la figura del seguro ambiental, que forma parte de la normativa obligatoria en la PBA 
y de todo el país y su cumplimiento es monitoreado por la OPDS. Dicho instrumento es controversial porque, en primer lugar, el seguro de caución no tendría la naturaleza jurídica de un seguro propiamente dicho, por lo que con su contratación no se estarían satisfaciendo los requisitos legales.

En segundo lugar, no existe traslación del riesgo. En el caso de producirse el daño ambiental, el sujeto dañoso deberá correr con los gastos de remediación y la caución obra solo como una garantía para el beneficiario - que es el Estado- de que la suma asegurada estará disponible y será afectada a las tareas de recomposición hasta alcanzar el monto asegurado.

En tercer lugar, la normativa impone la obligación de llevar a cabo la remediación por medio de los sujetos indicados por el asegurador en la póliza.

En cuarto lugar, en ocasiones el patrimonio de las aseguradoras sería inferior al del tomador. Además, es conveniente analizar si la actividad desarrollada por el establecimiento está obligada a contratar el seguro ambiental previsto en el Artículo 22 de la Ley General del Ambiente, de acuerdo con la normativa que se aplique según la jurisdicción donde se ubique el establecimiento.

El grado de incertidumbre jurídica creada por la reciente resolución judicial, que suspende la vigencia de normas dictadas durante el año 2012, ubica a los usuarios y operarios del sistema en extrema fragilidad. Más allá de la discrepancia en cuanto a si las normas de 2012 están suspendidas o no, atento lo que dispuso la Corte Suprema en 2014 y la Resolución 999/2014, a la fecha solo se comercializan en el mercado los seguros de caución.

Esto es porque la regulación del régimen de seguros (vigente y suspendido) es llevada a cabo por un entramado de resoluciones y decretos dictados por distintos organismos de Gobierno, con una profusa y confusa regulación en distintas direcciones, fruto de los distintos intereses que mueve. A ello se suma que es mediante una decisión judicial, solicitada por una organización ambiental, se suspenden las modificaciones establecidas al régimen anterior.

Otro de los instrumentos controversiales es la certificación voluntaria de cualquier organización. Las normas internacionales no pueden reemplazar, transformar o modificar de alguna forma la responsabilidad del Estado de actuar por el interés público y tampoco pretenden abordar cuestiones que solo pueden resolverse por medio de las instituciones políticas. 
Avanzar en la consolidación de una política ambiental requiere garantizar el fortalecimiento del OPDS, la implementación de los instrumentos de gestión ambiental vigentes, de las leyes y regulaciones y que se pueda avanzar en institucionalizar otros.

\section{Referencias}

Argentina, Congreso Nacional. (1993). Ley Provincial 11459, radicación industrial. Argentina, Congreso Nacional. (1995a). Ley 11723, Ley integral del medioambiente $y$ de los recursos naturales.

Argentina, Congreso Nacional. (1995b). Ley Provincial 11720, residuos especiales. Argentina, Congreso Nacional. (2002). Ley General del Ambiente, Ley 25675. Argentina, Congreso Nacional. (2012). Decreto 1638/12, seguro ambiental. Argentina, Corte Suprema de Justicia. (2012). Recurso de hecho deducido por la demandada en la causa Fundación Medio Ambiente c/ EN-PEN- dto. 1638/12-SSNresol. $37.160 \mathrm{~s} /$ medida cautelar autónoma.

Argentina, Secretaría de Ambiente y Desarrollo Sustentable. (2014). Resolución 999/2014, conformidad ambiental.

Argentina, Poder Ejecutivo Provincial. Decreto 23 de 2007.

Argentina, Secretaría de Ambiente y Desarrollo Sustentable de la Nación. (2011). Resolución 481/11, política ambiental.

Argentina, Superintendencia de Seguros de la Nación. (2012). Resolución 37160/12, seguro ambiental.

Argerich, A. (2009). Administración y desarrollo sustentable. Catamarca: Editorial Científica Universitaria.

Cáceres, V (2013). Aportes para el debate crítico: La mercantilización de la naturaleza y la contaminación. Delos. Desarrollo Local Sostenible, 6(18), 1-12.

Cáceres, V. (2014). El gasto público ambiental de la provincia de Buenos Aires, Argentina (1997-2012). Abra, 34(49), 1-27.

Coase, R. (1994). La empresa, el mercado y la ley. Madrid: Alianza.

Forcinito, K. (2010). Aproximación al estudio del pensamiento económico predominante en la Argentina desde los años setenta: la recepción doméstica del pensamiento neoliberal. Recuperado de http://www.aahe.fahce.unlp.edu.ar/jornadas-de-historiaeconomica/xxii-jornadas-de-historia-economica-rio-cuarto-2010/ponencias/ forcinito.pdf/view 
Instituto Nacional de Estadísticas y Censos. (2010). Censo nacional de población, hogares y viviendas de 2010. Censos del bicentenario. Resultados definitivos. Serie B $N^{\circ}$ 2. Tomo 1. Recuperado de http://www.censo2010.indec.gov.ar/archivos/ censo2010_tomo1.pdf

Isuani, F. (2010). Los caminos de la debilidad estatal. Capacidades estatales y politicas públicas. Una mirada desde el proceso de configuración de los instrumentos de politicas públicas. El caso de la política del agua en la Provincia de Buenos Aires (19922008). (Tesis doctoral). Recuperado de http://repositorio.flacsoandes.edu.ec/ bitstream/10469/2989/2/TFLACSO-2010FJI.pdf

Isuani, F. (2013). Institucionalidad Ambiental en la Provincia de Buenos Aires (19832011). Desafiosa la coherencia, integralidad y coordinación. Recuperado de http:// www.ungs.edu.ar/ms_ico/wp-content/uploads/2014/10/Pol\%C3\%ADticaAmbiental-en-la-Provincia-de-Buenos-Aire-Isuani-20131.pdf

Lloret, E. (2011). El principio preventivo y precautorio en el Derecho Ambiental. ¿A qué principio responde la evaluación de impacto ambiental? Recuperado de: http:// dialnet.unirioja.es/servlet/articulo?codigo $=4283316$

Lozano Cutanda, B. (2007). Derecho Ambiental Administrativo. Madrid: Dykinson. Menéndez, A. (2014). Derecho Ambiental. En W. Volkheimer, L. Scafati y D. Melendi (eds.), Breve enciclopedia del ambiente. Recuperado de http://www.cricyt. edu.ar/enciclopedia/terminos/DerAmb.htm

Minaverry, C. (2014). La relación entre la protección de los recursos hídricos y de los bosques. Situación legal en Argentina y en Uruguay. (Investigación en curso, 2014-2016). Departamento de Ciencias Sociales de la Universidad Nacional de Luján, Argentina.

Minaverry, C. y Gally, T. (2013). Las normas voluntarias SA 8000 e ISO 26.000 sobre responsabilidad social y su importancia ante la debilidad del Derecho. Ars Boni et Aequi, 9(2), 257-276.

Monsalve P. (2015). Enfoques económicos y representaciones sociales de la problemática ambiental desde una perspectiva crítica. (Investigación inédita). Instituto de Desarrollo Humano de la Universidad Nacional de General Sarmiento, Argentina. OPDS. (2010). Resolución 165/10, modificada a su vez por la Resolución 186/12 del 28/09/2012), seguro ambiental.

OPDS. (2015). Evaluación de impacto ambiental. Listado. Recuperado de http://www. opds.gba.gov.ar/EIA/EIA_mostrartodos_conbuscar.php

Pearce, D. y Turner, F. (1995). Economía de los recursos naturales y ambientales. Madrid: Colegio de Economistas de Madrid.

Pengue, W. (2008). Fundamentos de Economía Ecológica. Buenos Aires: Kaicron.

Pigou, A. (1950). The Economics of Welfare. Londres: MacMillan. 
Preiss, O. y Roca, S. (2006). Reestructuraciones locales en El Chañar a partir de la expansión de inversiones en la agroindustria. Recuperado de http://labuenafruta. com.ar/norpatagonia/reestructuraciones-locales-en-el-cha\%C3\%B1 ar-partirde-la-expansi\%C3\%B3n-de-inversiones-en-la-agr

Valenzuela Fuenzalida, R. (2010). El Derecho Ambiental. Presente y pasado. Santiago: Jurídica de Chile.

Williams, R. (2000). Marxismo y literatura. Barcelona: Península. 